\title{
Diffusion in membranes: Toward a two-dimensional diffusion map
}

\author{
Laura Toppozini ${ }^{1, \text { a }}$, Victoria Garcia-Sakai ${ }^{2}$, Robert Bewley ${ }^{2}$, Robert Dalgliesh $^{2}$, Toby Perring ${ }^{2}$ \\ and Maikel C. Rheinstädter ${ }^{1, b}$ \\ ${ }^{1}$ Department of Physics and Astronomy, McMaster University, Hamilton, ON, Canada \\ ${ }^{2}$ ISIS, Rutherford Appleton Laboratory, Didcot, UK
}

\begin{abstract}
For decades, quasi-elastic neutron scattering has been the prime tool for studying molecular diffusion in membranes over relevant nanometer distances. These experiments are essential to our current understanding of molecular dynamics of lipids, proteins and membrane-active molecules. Recently, we presented experimental evidence from X-ray diffraction and quasi-elastic neutron scattering demonstrating that ethanol enhances the permeability of membranes. At the QENS 2014/WINS 2014 conference we presented a novel technique to measure diffusion across membranes employing 2-dimensional quasielastic neutron scattering. We present results from our preliminary analysis of an experiment on the cold neutron multi-chopper spectrometer LET at ISIS, where we studied the self-diffusion of water molecules along lipid membranes and have the possibility of studying the diffusion in membranes. By preparing highly oriented membrane stacks and aligning them horizontally in the spectrometer, our aim is to distinguish between lateral and transmembrane diffusion. Diffusion may also be measured at different locations in the membranes, such as the water layer and the hydrocarbon membrane core. With a complete analysis of the data, 2-dimensional mapping will enable us to determine diffusion channels of water and ethanol molecules to quantitatively determine nanoscale membrane permeability.
\end{abstract}

\section{Introduction}

Diffusion of water in membranes occurs over small length scales and time scales making it well suited for study using quasi-elastic incoherent neutron scattering (QENS). With the latest generation of neutron time-offlight and backscattering spectrometers with large detector coverage, high pixel resolution, and increasingly powerful neutron sources, it is now possible to simultaneously measure scattering from both lateral and transmembrane diffusion of molecules in membranes. By using oriented membranes, dynamics are collected simultaneously in all detector pixels and can be assigned to in-plane and out-of-plane wave vectors, $\mathrm{q}_{\|}$and $\mathrm{q}_{z}$, respectively. The result of such an experiment is a 2-dimensional mapping of lateral and transmembrane diffusion coefficients at different length scales to determine a complete picture of molecular dynamics.

Prior to this novel use of position-sensitive detectors, quasi-elastic incoherent neutron scattering experiments to measure self-diffusion of 2-dimensional systems were done using vertically oriented samples [2]. Measurement of QENS of 2-dimensional systems (e.g. clays, graphite, liquid crystals, and solid-supported lipid membranes) typically involves a geometry where the sample (whose layers are parallel to the plane of the substrate) lies vertically and is held at an angle with respect to the incident neutron beam. The neutrons detected at a

\footnotetext{
a e-mail: toppozl@mcmaster.ca

b e-mail: rheinstadter@mcmaster.ca
}

scattering angle equal to $90^{\circ}$ from a sample oriented at an angle of $45^{\circ}$ to the incident neutrons measures the momentum transfer vector $\mathrm{q}_{z}$, corresponding to scattering from diffusive motion normal to the layers [2]. By rotating this vertically oriented sample to $135^{\circ}$ with respect to the incident neutron beam, detecting the neutrons $90^{\circ}$ from the incident beam measures the momentum transfer vector $\mathrm{q}_{\|}$, corresponding to scattering from diffusive motion in the plane of the layers [2]. This technique serves to find the quasi-elastic scattering one q-value at a time using a point detector, which can be very time consuming. However, for large area detector banks the additional scattering information is a mixture of the two momentum transfer vectors $\mathrm{q}_{\|}$and $\mathrm{q}_{\perp}$ and thus cannot give purely perpendicular or parallel diffusive information.

Many studies have used the previously described, vertical sample orientation technique which is used to characterize the anisotropic diffusive motion of lipids in bilayers including the seminal works by Pfeiffer et al. [3] who determined the rotational, vibrational and diffusive motions of lipids in hydrated single-component bilayers and König et al. [4], who determined the relationship between temperature and hydration on the rotational, vibrational, and diffusive motions of lipids in single-component membranes. More recently, the aforementioned technique has been used on more complex systems, for instance introducing a peptide in a membrane consisting of two lipid species [5], showing the effect of this peptide on lipid dynamics. With the longstanding use of this vertical sample orientation technique we are hopeful that the novel horizontal sample

This is an Open Access article distributed under the terms of the Creative Commons Attribution License 4.0, which permits unrestricted use, distribution, and reproduction in any medium, provided the original work is properly cited. 


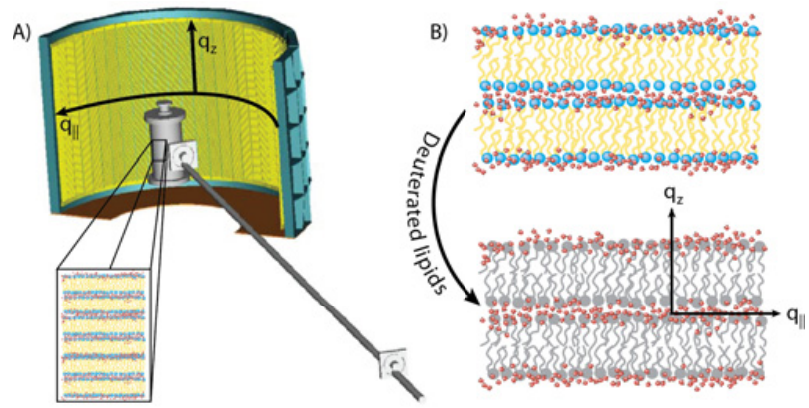

Figure 1. Schematics of the experimental setup. A) Schematic of the LET (Low Energy Transfer) instrument at ISIS (adapted from [1]). In the sample can, our experiment housed horizontal, solid-supported, stacked bilayers of deuterated DMPC hydrated to $\sim 100 \%$ with $\mathrm{H}_{2} \mathrm{O}$. The position sensitive detectors allow for data collection of both lateral $\left(\mathrm{q}_{\|}\right)$and transmembrane $\left(\mathrm{q}_{z}\right)$ diffusion. B) By using deuterated lipids, we are sensitive to the incoherent scattering of the water in the membrane and the selfdiffusive motion of the water molecules.

orientation technique we present here has the potential to improve diffusion measurements in this field and allow new types of samples to be measured (e.g. that cannot have vertical orientation). Here, we look at the self-diffusion of water in a lipid membrane, which encounters anisotropic motion due to the vastly different environments as viewed from the in-plane and out-of-plane directions.

We recently presented experimental evidence from Xray diffraction and quasi-elastic neutron scattering that ethanol enhances the permeability of membranes [6]. We determined the location and partitioning of ethanol molecules in single-component lipid bilayers. We found that ethanol induces an increase of solvent molecules in the hydrophobic core of the membrane, however the use of $\mathrm{X}$-rays does not allow for differentiation between solvent molecules, solely based on the electronic distribution. The presence of water in the hydrophobic membrane core is indicative of an increase in permeability of the bilayer, whereas additional ethanol molecules in the core would point to ethanol's function as an anesthetic due to an increase in lateral pressure from the density increase inside the core.

More recently, we measured quasi-elastic incoherent neutron scattering from water hydrating stacked, solidsupported, oriented lipid membranes at the Low Energy Transfer (LET) instrument at ISIS, shown in Fig. 1A. Using this instrument, our intention is to find the selfdiffusion of water and in pure water and water-ethanol mixtures $(5 \mathrm{~mol} \%)$ along and across membranes by employing a novel 2-dimensional quasi-elastic scattering technique. With the use of selective deuteration, we were able to isolate the diffusive motion of the water molecules in hydrated membranes, as depicted in Fig. 1B. LET covers three orders of magnitude in time: from $\sim 0.5$ picoseconds to almost 1 nanosecond; this timescale compliments our previous backscattering results [6]. The data and current, yet partial, analysis from this experiment on the cold neutron multi-chopper spectrometer LET at ISIS were presented at the QENS 2014/WINS 2014 conference in Autrans, France.

\section{Sample preparation}

All samples used in this experiment consisted of stacked, solid-supported, and highly oriented singlecomponent lipid bilayers. Bilayers were synthesized by dissolving chain deuterated 1,2-dimyristoyl-d54-snglycero-3-phosphocholine (DMPC-d54) in a mixture of $50 \%$ 2,2,2-trifluoroethanol and 50\% chloroform at a concentration of $15 \mathrm{mg} / \mathrm{mL}$. An aliquot of $1 \mathrm{~mL}$ was deposited onto clean, 2-diameter silicon wafers. Due to solvent evaporation, the lipids self-assemble into bilayers whose plane is that of the wafer, ensuring a high degree of orientation. The bilayers were subsequently annealed to ensure the highest homogeneity. Eighteen wafers were stacked into an aluminum sample can where two $300 \mu \mathrm{m}$ spacers sat between each wafer, tension was used to keep the wafers in place; aluminum foil placed between the wafers and sample can lid served this purpose.

Ethanol's volatility is a particular challenge for scattering experiments. In previous studies, membranes were immersed in a water-ethanol solution to ensure a specific concentration of ethanol molecules in the bilayers $[6,9,10]$. High absorption and background in the neutron spectra is detected as a result of the large proportion of solvent. In this experiment, the bilayers were hydrated with $\mathrm{H}_{2} \mathrm{O}$ from the vapour phase. Due to low scattering of the sample (typically $\sim 10 \%$ ), we did not observe direct evidence of multiple scattering events and therefore, we assume all scattering is solely from the sample. The bilayers were hydrated through vapour from water and water-ethanol solutions by placing solvent-saturated filter paper above aluminum foil. Data were taken while the membranes were in the fluid phase, at a temperature of $\mathrm{T}=293 \mathrm{~K}\left(30^{\circ} \mathrm{C}\right)$. The neutron scattering experiments were carried out at the instrument LET at ISIS, the pulsed neutron and muon source at the Rutherford Appleton Laboratory in Oxfordshire, UK. Incident neutron energies were: $0.64,0.96,1.62,3.28$, and $9.8 \mathrm{meV}$ (corresponding to $\lambda=2.89-11.3 \AA$ ).

\section{Results and discussion}

Ethanol is known to increase the fluidity of membranes and increase their permeability; however, the exact molecular mechanism is still unclear leaving many unanswered questions about such a ubiquitous molecule. Potential mechanisms include changes in lateral pressure that may affect protein function and binding sites and increases in membrane permeability to change electrostatic potentials which may affect signalling. Some questions that we intend to answer with these data are, for example, what is the effect of ethanol on the dynamics of lipid bilayers? What is its impact on membrane permeability?

Taking advantage of the unique features of LET allows insight into the molecular dynamics of lipids, ethanol and water in lipid membranes in-situ, under physiological conditions. The high-flux and the large position-sensitive detectors of LET, in combination with horizontal, oriented membrane samples, gives us the potential to study not only in-plane, but transmembrane dynamics of the different molecular contributions separately but simultaneously. We used selective deuteration of ethanol, water, and lipids 


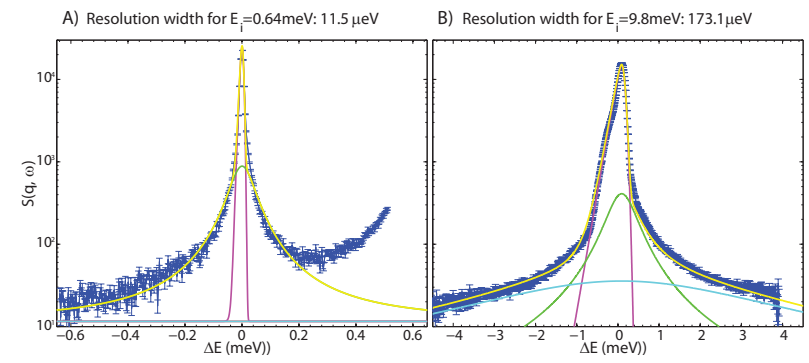

Figure 2. Shown is a fit to a spectrum for the first $\mathrm{q}_{z}$ slice in a sample consisting of deuterated lipids (DMPC-d54) hydrated with $\mathrm{H}_{2} \mathrm{O}$. The bin was centred at $\mathrm{q}_{||}=0.36 \AA^{-1}$. The magenta curve is the central Gaussian resolution function. The green and cyan peaks are Lorenztian functions and correspond to a slow and fast motion, respectively. On the right side of each spectra we observe an inelastic peak most likely due to collective dynamics of the lipid membranes as previously observed $[7,8]$. A) The energy of the incident neutrons is $0.64 \mathrm{meV}$ resulting in a width of the Gaussian peak of $11.5 \mu \mathrm{eV}$. B) The energy of the incident neutrons is $9.80 \mathrm{meV}$ with a resolution width of the Gaussian peak of $173.1 \mu \mathrm{eV}$.

to unambiguously assign the observed dynamics to the different molecular components. In the following, we limit the discussion to the current state of analysis and will solely address the lateral self-diffusion of water in a hydrated lipid bilayer system. By using deuterated lipids (DMPC-d54) and protonated water $\left(\mathrm{H}_{2} \mathrm{O}\right)$, we collect the quasi-elastic incoherent neutron scattering from the sample which allows for the characterization of the self-diffusion of water in a lipid bilayer environment.

Quasi-elastic neutron scattering data were taken with 5 different incident neutron energies, $\mathrm{E}_{i}: 0.64 \mathrm{meV}$, $0.96 \mathrm{meV}, 1.62 \mathrm{meV}, 3.28 \mathrm{meV}$, and $9.80 \mathrm{meV}$. By fitting each spectrum with two Lorentzians, we assert that two diffusive processes occur: slower diffusive motion (the narrow Lorentzian with width $\Gamma_{1}$ ) and faster diffusive motion (the broad Lorentzian with width $\Gamma_{2}$ ), each deconvolved with the instrumental resolution $(R)$ :

$$
\begin{aligned}
S\left(q_{\|, \perp}, \hbar \omega\right) & =R \otimes\left[\delta(\hbar \omega)+L\left(\Gamma_{1}\right)+L\left(\Gamma_{2}\right)\right] \\
& =R \otimes\left[\delta(\hbar \omega)+\frac{1}{\pi} \frac{\frac{1}{2} \Gamma_{1}}{(\hbar \omega)^{2}+\left(\frac{1}{2} \Gamma_{1}\right)^{2}}\right. \\
& \left.+\frac{1}{\pi} \frac{\frac{1}{2} \Gamma_{2}}{(\hbar \omega)^{2}+\left(\frac{1}{2} \Gamma_{2}\right)^{2}}\right] .
\end{aligned}
$$

Each quasi-elastic spectrum was fit with a resolution (asymmetric Gaussian profile) and two Voigt profiles, as shown in Fig. 2. The corresponding motion for the lateral diffusion was modeled as Brownian diffusion by using the Lorentzian widths of the Voigt profile; widths (FWHM, $\Gamma$ ) plotted against the square of the central q-value for that $\mathrm{q}_{\|}$ bin were well fit to a straight line, as shown in Fig. 3. The diffusion constant for Brownian diffusion is determined from the slope fit to the points from a Lorentzian width vs. $\mathrm{q}_{\|}^{2}$ plot using the following:

$$
\Gamma=2 \hbar D q_{\|}^{2}
$$

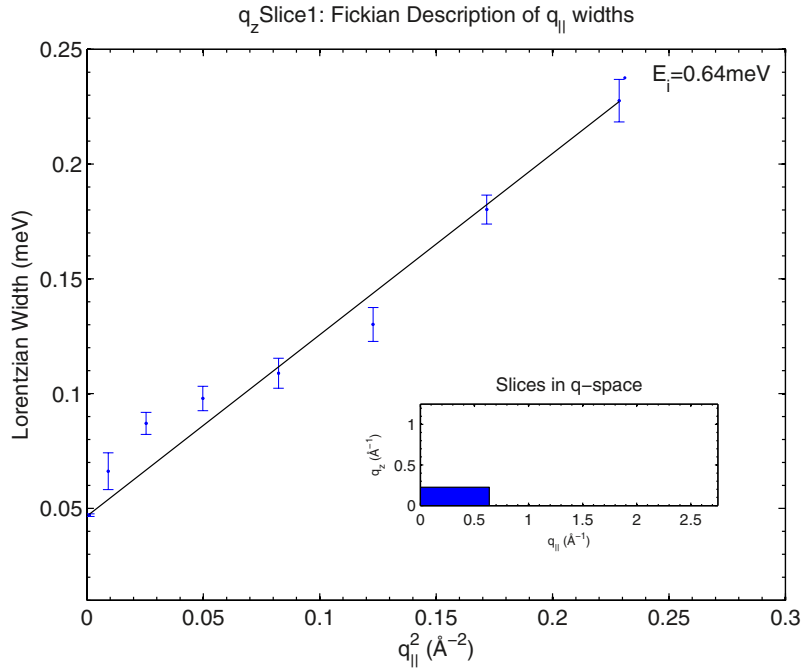

Figure 3. Example plot for Lorentzian width vs. $\mathrm{q}_{\|}^{2}$ for a incident neutron energy of $0.64 \mathrm{meV}$. The region of q-space covered can be seen in the inset. The data are binned along $\mathrm{q}_{\| \mid}$and each bin is a spectrum which is fit with a Voigt function. For each spectrum, the Lorenztian width from the slow-motion peak is plotted against the square of the center of each bin. The resultant diffusion constant for this lateral slice is $D_{\|}=(5 \pm 1) \times 10^{-9} \mathrm{~m}^{2} / \mathrm{s}$.

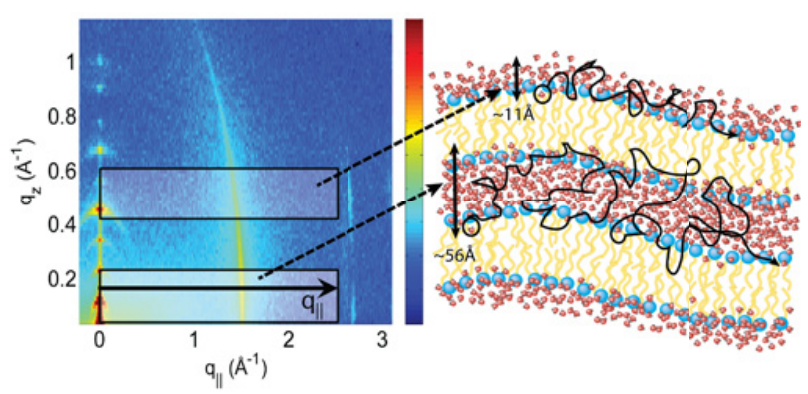

Figure 4. Schematic of lateral diffusion ranges of water in a membrane. An exemplary X-ray diffraction plot (left) of DMPC (plotted as $\mathrm{q}_{z}$ against $\mathrm{q}_{\|}$) shown to illustrate the $\mathrm{q}$ ranges of the data-slicing of the LET data. The plot is taken from the Laboratory of Lipid and Protein Dynamics at McMaster University. On the right, a schematic of real-space trajectory of a water molecule. Each lateral diffusion covers a corresponding $\mathrm{q}_{z}$ range, depicted.

The position-sensitive $\mathrm{He}^{3}$ detectors have the potential to record a 2-dimensional mapping of the quasi-elastic neutron scattering. The pixels from this map were sliced and binned into sections in the two directions ( $z$-direction and $x-y$-plane). For the lateral diffusion slicing, we started by slicing the $\mathrm{q}_{z}$ direction into five large slices of equal width over the entire angular range of $\pm 30^{\circ}$ of the data, as depicted in Fig. 4. Each slice was then binned into 10 equal-width bins along $\mathrm{q}_{\|}$. It should be noted that though the number of bins is 10 , often only 8 spectra can be fit due to higher bins having spectra which do not contain a full lineshape, and they can therefore not be associated with a Lorenztian width, as in Fig. 3. Each lateral binning in $q_{\|}$was integrated over a certain range in $\mathrm{q}_{z}$, as depicted in Fig. 4. The measurements covered a lateral $q_{\|}$-range from $0.03 \AA^{-1}$ (for the lowest energy 
neutrons of $0.64 \mathrm{meV}$ ) up to $2.37 \AA^{-1}$ for the highest energy neutrons of $9.80 \mathrm{meV}$. The corresponding diffusion processes are tentatively pictured in the Fig. 4.

Using this slicing scheme, we can tentatively assign a lateral diffusion constant of $\mathrm{D}_{\|}=(5 \pm 1) \times 10^{-9} \mathrm{~m}^{2} / \mathrm{s}$ to the first $\mathrm{E}_{i}=0.64 \mathrm{meVq}_{z}$ slice covering a real-space $z$-range of up to $56 \AA$ and $\mathrm{D}_{\|}=(4 \pm 2) \times 10^{-9} \mathrm{~m}^{2} / \mathrm{s}$ to the second $\mathrm{q}_{z}$ slice covering a real-space $z$-range of up to $28 \AA$. It has been previously reported by Krynicki et al. [11] that the diffusion constant of bulk water at standard pressure and a temperature of $298.2 \mathrm{~K}$ is $\mathrm{D}=$ $2.30 \times 10^{-9} \mathrm{~m}^{2} / \mathrm{s}[11]$ in their paper utilizing the nuclear magnetic resonance spin echo technique. Teixeira et al. and Qvist et al. have published results from quasi-elastic incoherent neutron scattering from bulk water at a variety of temperatures where they found that two relaxation processes were associated with the quasi-elastic peak $[12,13]$, which is how we have chosen to analyze our lateral diffusion data thus far. Qvist et al.'s diffusion constants as a result of experiment and molecular simulations at $297.1 \mathrm{~K}$ are $2.16 \times 10^{-9} \mathrm{~m}^{2} / \mathrm{s}$ [13] and $2.20 \times 10^{-9} \mathrm{~m}^{2} / \mathrm{s}$ [13], respectively. We can at this point not comment on the difference between our diffusion constants with respect to bulk water. We note, however, that our values are in good agreement with a recent simulation study, where a diffusion constant of $3.7 \times$ $10^{-9} \mathrm{~m}^{2} / \mathrm{s}$ was reported for membrane-hydrated water molecules [14].

Transmembrane diffusion constants of water molecules in single-component lipid bilayers and in the presence of ethanol molecules will be determined in the future, once the data analysis is completed. Analysis will be much the same as the lateral diffusion analysis; by slicing the data in the $\mathrm{q}_{\|}$axis and binning along the $\mathrm{q}_{z}$ axis.

we have the potential to find the self-diffusion of water across the membrane. We are hopeful that this analysis will yield a way to find different mechanisms by which water or other small molecules permeate the bilayer. By analyzing the different detector slices, a 2-dimensional map of diffusion constants along and across the bilayers has the potential to be determined. In particular, such a map will be important to determine potential 'diffusion channels' in membranes for certain types of molecules.

\section{References}

[1] http://www.isis.stfc.ac.uk/instruments /let/science/let-science-6442.html

[2] M. Bée, Quasielastic Neutron Scattering: Principles and Applications in Solid State Chemistry, Biology and Materials Science (Taylor \& Francis, 1988), ISBN 0-852-74371-8

[3] W. Pfeiffer, T. Henkel, E. Sackmann, W. Knorr, Europhys. Lett. 8, 201 (1989)

[4] S. König, W. Pfeiffer, T. Bayerl, D. Richter, E. Sackmann, J. Phys. II France 2, 1589 (1992)

[5] A. Buchsteiner, T. Hauß, S. Dante, N. Dencher, Biochim. Biophy. Acta 1798, 1969 (2010)

[6] L. Toppozini, C.L. Armstrong, M.A. Barrett, S. Zheng, L. Luo, H. Nanda, V. Garcia Sakai, M. Rheinstädter, Soft Matter 8, 11839 (2012)

[7] M.C. Rheinstädter, K. Schmalzl, K. Wood, D. Strauch, Phys. Rev. Lett. 103, 128104 (2009)

[8] C.L. Armstrong, M.A. Barrett, A. Hiess, T. Salditt, J. Katsaras, A.C. Shi, M.C. Rheinstädter, Eur Biophys J 41, 901 (2012)

[9] M.D. Kaye, K. Schmalzl, V.C. Nibali, M. Tarek, M.C. Rheinstädter, Phys. Rev. E 83, 050907 (2011)

[10] J. Yang, C. Calero, J. Marti, Journal of Chemical Physics 140, 104901(1) (2014)

[11] K. Krynicki, G. C.D., S. D.W., Faraday Discuss. 66, 199 (1978)

[12] J. Teixeira, M.C. Bellisent-Funel, S. Chen, A. Dianoux, Phys. Rev. A 31, 1913 (1985)

[13] J. Qvist, H. Schober, B. Halle, J. Chem. Phys. 134, 144508(1) (2011)

[14] Y. Von Hansen, S. Geckle, R.R. Netz, Phys. Rev. Lett. 111, 118103(1) (2013) 